\title{
Drug utilization study in neonatal intensive care unit at tertiary care hospital
}

\author{
Farshad Namdarifar ${ }^{1}$ (), Shahrzad Raouf ${ }^{1 *}$ (D), Emad Malekpour Shahraki ${ }^{1} \mathbb{D}$, N.B. Sridhara Murthy ${ }^{1}$
}

\section{SUMMARY}

OBJECTIVE: Neonates are more susceptible to drug interactions and adverse effects, and special care should be taken when prescribing medication to them. This study aimed to investigate drug usage in the neonatal intensive care unit of a tertiary care hospital.

METHODS: This prospective observational study was conducted on 98 patients at the Apollo tertiary care hospital (Bannerghatta, Bangalore, India) in a period of 6 months. The most common indications for neonatal intensive care unit admission, average number of drugs per patient, the most frequently used medication, distribution of patients based on the birth procedure, and possible drug interactions were collected from patient profiles. RESULTS: Among the patients, $52 \%$ were males and $48 \%$ were females. Notably, $38 \%$ of patients were preterm, $60 \%$ were term, and only $2 \%$ were post-term. Also, $80.6 \%$ were born by cesarean section and $19.4 \%$ were born by normal vaginal delivery. The highest mean of drug use was in the patient of 1,000-1,500 g (8.06 per patient). Preterm was the most frequent indication for admission in neonatal intensive care unit, followed by hyperbilirubinemia and then respiratory distress syndrome. The most frequently used medication was vitamin K (99\%) and antibiotics followed by dextrose. In different types of antibiotics, amikacin (41\%), cefoperazone+sulbactam (35\%), cephalosporin (1\%), ceftriaxone (0.7\%), and amoxicillin (0.3\%) were commonly administered. There were some possible interactions, such as aminoglycoside with furosemide and calcium gluconate.

CONCLUSION: Premature birth and resulting low birth weight were the main reasons for drug prescription. High administration of antibiotics is probably an area of concern and should be seriously considered.

KEYWORDS: Drug utilization. NICU. Infant.

\section{INTRODUCTION}

The drug utilization studies are used as effective tools in drug administration by the physician and hospital regarding audit and cost analysis and can recognize rational drug administration. With the rise of new drugs in the market, variations of prescribing pattern and administration of drugs, new adverse effects, and the concerns regarding the cost of these drugs, the importance of drug utilization studies is becoming increasingly apparent ${ }^{1,2}$.

The drug utilization studies apply epidemiological methods to study the clinical use of drugs in populations ${ }^{3}$. These studies in newborns may be used to identify the major therapeutic problems. Although rational drug therapy is important for all individuals being treated with drugs, it is of paramount importance for neonates. Therefore, it is necessary to analyze the pattern of drug use in neonatal units and to identify therapeutic classes that should be prioritized for future research ${ }^{4,5}$.

A neonatal intensive care unit (NICU) is a highly specialized unit that provides high-quality skilled care to premature, low birth weight, or critically ill newborn. Apart from facilities for continuous clinical and biochemical monitoring and life support systems, the neonatal intensive care management involves the use of a wide range of medications with well-defined and specified therapeutic objectives ${ }^{6}$. Because of the immaturity of various organ functions, such as kidney, liver, and gastric motility, the neonates may show pharmacodynamics and pharmacokinetic variations. Furthermore, they have a rapidly changing body surface area and weight, a rapidly developing system of drug absorption, metabolism, and excretion, and an inability to communicate with the provider, making them more susceptible to adverse drug reactions and irrational drug use $e^{6,7}$.

The most common reasons for NICU admission are prematurity, respiratory distress syndrome (RDS), sepsis or infection, hypoglycemia, perinatal depression, and maternal chorioamnionitis ${ }^{8,9}$. Fluid therapy, antibiotics, vitamin $\mathrm{K}$, vitamin $\mathrm{D}$, calcium, and heparin are among the most common medicine used in $\mathrm{NICU}^{10,11}$.

There are few drug utilization studies on NICU admitted patients. Neonate is a group of patients who are more susceptible to drug interactions and adverse effects, and special care should be taken when prescribing medication to them. This study aimed to investigate drug usage in NICU of a tertiary care hospital and possible drug interactions.

${ }^{1}$ Rajiv Gandhi University of Health Sciences, Oxbridge College of Pharmacy, Department of Pharmacy - Bangalore, India.

*Corresponding author: Shahrzad.raouf@yahoo.com

Conflicts of interest: the authors declare there is no conflicts of interest. Funding: none.

Received on September 26, 2021. Accepted on December 05, 2021. 


\section{METHODS}

This prospective observational study was conducted on 98 patients at the Apollo tertiary care hospital (Bannerghatta, Bangalore, India) in a period of 6 months (from December 2019 to May 2020). Patients of either gender, who aged below 6 months, and who were admitted to NICU were included in the study. All patients who were admitted other than NICU and neonates who were not receiving any medications other than vaccines were excluded from the study. Full details of the cases, including gender, age, diagnosis, and drug therapy, were noted in the data collection form. All the patients were monitored until they shifted from NICU. The most common indications for NICU admission, average number of drugs per patient, the most frequently used medication, distribution of patients based on the birth procedure, and possible drug interactions were collected from patient profiles. Gestational age was defined as follows: preterm: born before week 37 of pregnancy; term: born between weeks 37 and 40 of pregnancy; and post-term: born after week 40 of pregnancy. The drug-drug interactions were analyzed using www.drugs.com as the resource. The study was approved by the Institutional Ethics Committee - Biomedical
Research ("IEC-BMR") of Apollo hospital. The data were analyzed using SPSS version 16.0. and Microsoft Excel. The results were expressed in terms of percentages and numbers.

\section{RESULTS}

Among 100 patients enrolled in the study, 48 were females and 52 were males. Out of 100 patients, 60 were term, 38 were preterm, and 2 were post-term. In all 100 patients, preterm patients were having the most hospital stay with a mean of 5.94 days, and post-term patients were having least hospital stay with a mean of 3.5 days. Based on birth weight, the highest mean of drug use was 8.06 in the group of $1,001-1,500 \mathrm{~g}$, followed by 7.67 in the group of $1,501-2,000 \mathrm{~g}$. The least mean of drug use was 4.96 in the group of $\geq 4,000 \mathrm{~g}$ (Table 1 ).

Out of 98 patients, 79 were born by vaginal delivery, and 19 were born by cesarean section. Among the patients, 54 were appropriate for gestation age (AGA), 42 were small for gestation age (SGA), and 2 were large for gestational age (LGA). In addition, in preterm patients, most of them were SGA and, in term patients, AGA patients were most frequent (Figure 1).

Table 1. Mean drug use in different birth weight groups.

\begin{tabular}{|l|c|c|c|c|c|c|c|}
\hline Weight $(\mathbf{g})$ & $\mathbf{1 , 0 0 1 - 1 , 5 0 0}$ & $\mathbf{1 , 5 0 1 - 2 , 0 0 0}$ & $\mathbf{2 , 0 0 1 - 2 , 5 0 0}$ & $\mathbf{2 , 5 0 1 - 3 , 0 0 0}$ & $\mathbf{3 , 0 0 1 - 3 , 5 0 0}$ & $\mathbf{3 , 5 0 1 - 4 , 0 0 0}$ & $>\mathbf{4 , 0 0 0}$ \\
\hline Mean of drug use (number) & 8.06 & 7.67 & 7.03 & 6.12 & 5.87 & 5.24 & 4.96 \\
\hline
\end{tabular}

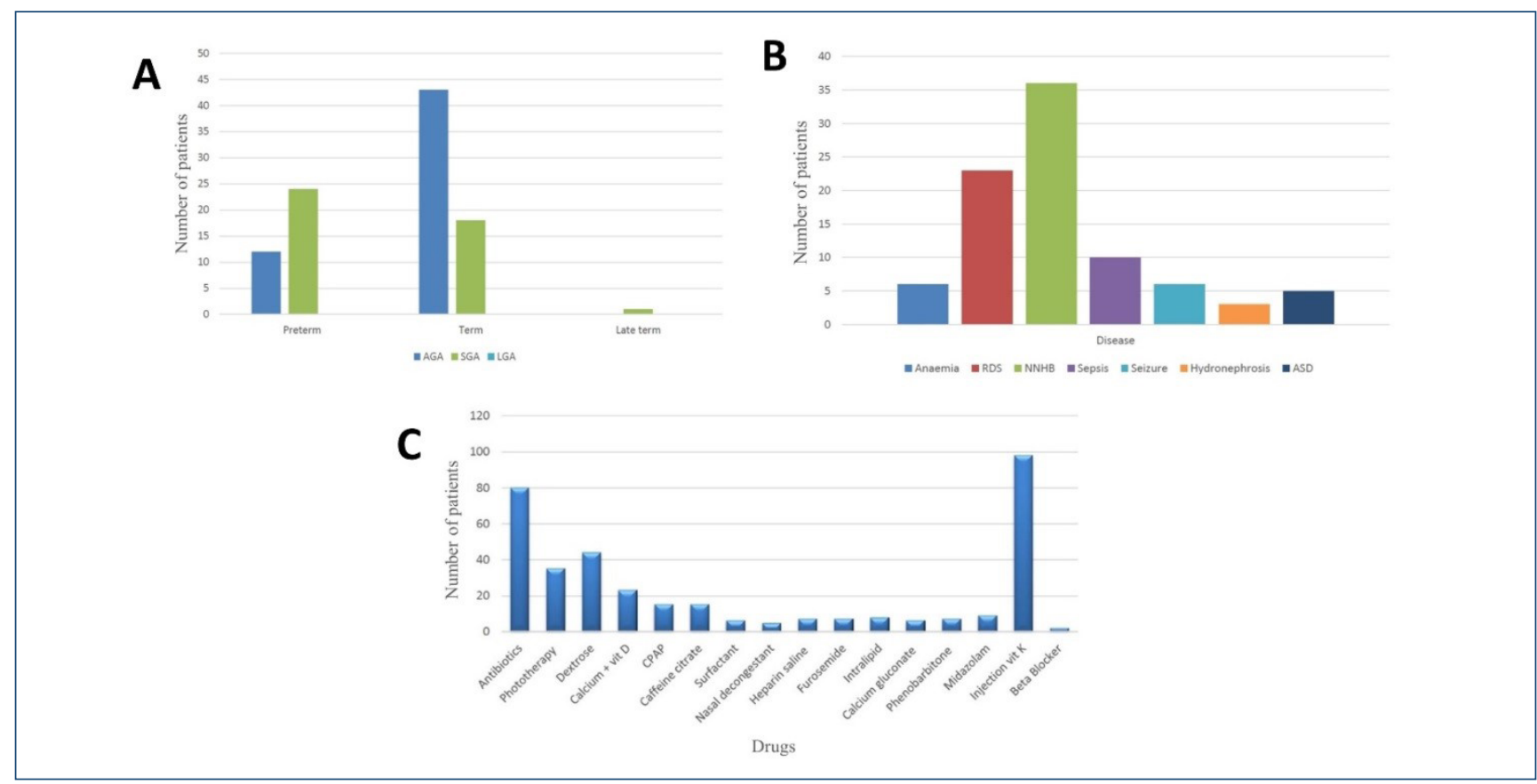

Figure 1. (A) Distribution of patients according to gestational age and birth weight. (B) Clinical indications for the neonatal intensive care unit admission and (C) different therapeutic class of drug prescribed. CPAP: continues positive airway pressure. 
Analysis of the clinical indication for admission at the NICU showed that neonatal hyperbilirubinemia (NHB) (36\%) followed by RDS (23 patients), sepsis (10 patients), anemia and sepsis (each 1 of the 6 patients), autism spectrum disorder (ASD) (5 patients), and hydronephrosis (3 patients) were the common clinical disorders (Figure 1B).

The most frequently used drugs in NICU were vitamin $\mathrm{K}$ and antibiotics [i.e., amikacin (41\%), cefoperazone+sulbactam (35\%), cephalosporin, ceftriaxone, and amoxicillin were the most commonly administered] followed by dextrose and phototherapy. Beta-blockers were the least frequently used drugs, followed by nasal decongestant (Figure 1C). Some possible drug interactions are also shown in Table 2.

\section{DISCUSSION}

Drug utilization study is an effective mechanism to identify individual variability in drug use and to promote interventions that will improve patient outcomes ${ }^{12}$. For the individual patient, the rational use of a drug implies the prescription of a well-documented drug at an optimal dose, together with the correct information, at an affordable price. Without the knowledge of how drugs are being prescribed and used, it is difficult to initiate a discussion on rational drug use or to suggest measures to improve prescribing habits. Information on the past performance of prescribers is the linchpin of any auditing system ${ }^{1}$. Neonates are a group of patients who are more susceptible to drug interactions and adverse drug reactions, and there are few studies on drug utilization pattern of these patients in
$\mathrm{NICU}^{4,13}$. This was a prospective study in a tertiary care hospital which was conducted for a period of 6 months to investigate the utilization pattern of drugs in NICU.

Data indicated that $52 \%$ of patients were males and $48 \%$ were females similar to the results of Kumbhar et al.'s ${ }^{14}(53.17 \%$ were males and $46.83 \%$ were females) and Choure et al.'s ${ }^{15}$ (54.3\% were males and $45 \%$ were females) studies. Among the patients, $38 \%$ were preterm, $60 \%$ were term, and only $2 \%$ were post-term. Unlike our results, Patel Brijal et al. ${ }^{16}$ carried out a study on 650 neonates in the Government Medical College and Hospital, Rajkot, in which $59.85 \%$ were preterm and $38.46 \%$ were term. Regarding the birth weight of patients, $54 \%$ were AGA, 43\% SGA, and only 2\% were LGA, according to the study by Schlaudecker et al. Distribution of patients based on the birth procedure showed that $80.6 \%$ were born by cesarean section and $19.4 \%$ were born by normal vaginal delivery, which is comparable with the study by Gonçalves AC de $\mathrm{S}$ et al. (39\% were vaginal delivery and $56.7 \%$ were cesarean section $)^{17}$.

The most frequent reasons for admission in NICU were preterm (39\%), followed by hyperbilirubinemia (37\%) and RDS (23\%), which is contrary to the study by Patel Brijal et al. (32\% sepsis, $27 \%$ RDS, and 25\% meconium aspiration syndrome $)^{16}$. These results indicate that prenatal care to prevent preterm delivery is one of the most important factors in reducing the rate of hospitalization and treatment costs.

Results showed that the average number of drugs per patient was 2.84. The most frequently used medication was vitamin $\mathrm{K}(99 \%)$ and antibiotics, followed by dextrose. The study by Neubert et al. ${ }^{18}$ has reported lower administration of vitamin

Table 2. Different antibiotics used and possible drug interactions.

\begin{tabular}{l|c|c|c|c|c}
\hline Antibiotics & Amikacin & Sulbactam+cefoperazone & Amoxicillin & Cephalosporin & Ceftriaxone \\
\hline Frequency & 33 & 28 & 3 & 10 & 6 \\
\hline Percentage & 41 & 35 & 0.3 & 1 & 0.7 \\
\hline
\end{tabular}

\begin{tabular}{l|c|c}
\hline \multicolumn{2}{c}{ Possible drug interactions } \\
\hline Drug 1 & Drug 2 & Effect \\
\hline Aminoglycoside & Furosemide & Increase risk of nephrotoxicity and ototoxicity \\
\hline Phenobarbitone & Triclofos (sedative) & Increase risk of sedation \\
\hline Benzodiazepine & Triclofos (sedative) & Increase risk of sedation \\
\hline Benzodiazepine & Aminoglycoside & Decrease sedation \\
\hline Dobutamine & Caffeine & Decrease serum potassium \\
\hline Dobutamine & Furosemide & Decrease effect of benzodiazepine calcium gluconate level \\
\hline Phenobarbitone & Benzodiazepine & Increase sedation \\
\hline Aminoglycoside & Calcium gluconate & Morphine \\
\hline Benzodiazepine & Dect aminoglycoside \\
\hline
\end{tabular}


$\mathrm{K}$ in neonates $(90.06 \%)$. Choure et al. ${ }^{15}$ conducted a study on 220 neonates in NICU of Government Medical College, Ambajogai, Maharashtra. Near to our results, the total number of drugs prescribed was 808 , and the average number of drugs per prescription was 3.6. However, the most frequently prescribed therapeutic class of drugs were antimicrobial agents (60.64\%), followed by vitamin K (26.7\%) and aminophylline (9.4\%). The average number of drug administration should be less as much as possible to reduce the chance of drug-drug interaction, and adverse effects of drugs, and the cost of treatment.

The results of this study showed that the infant's weight is a very influential factor in the amount of medication prescribed. Out of 98 patients, the highest mean of drug use was 8.06 and 7.67 in the patients of $1,000-1,500 \mathrm{~g}$ and $1,501-2,000 \mathrm{~g}$, respectively, and it was reduced to 4.96 in the patients of $>4,000 \mathrm{~g}$. Consistent with our results, Kumbhar et al. ${ }^{14}$ reported that the mean of drug use was 8.125 in $\leq 1,000 \mathrm{~g}$ birth weight neonates. Given that one of the main reasons for low birth weight is premature birth, prenatal care should be seriously considered.

In the present study, vitamin $\mathrm{K}$ was the most frequently used medication (99\%), and antibiotics were the next followed by dextrose. The study of Neubert et al. ${ }^{18}$ reported lower prescription of vitamin $\mathrm{K}$ in neonates $(90.06 \%)$. Vitamin $\mathrm{K}$ deficiency bleeding is of particular concern in neonates as they are born with low levels of vitamin $\mathrm{K}$. Current recommendations suggest single intramuscular administration of vitamin $\mathrm{K}$ as an effective, safe, and sustainable approach to prevent vitamin $\mathrm{K}$ deficiency bleeding.

Antibiotics were the second most prescribed drugs. In different types of antibiotics, amikacin and cefotaxime were the

\section{REFERENCES}

1. Shalini S, Ravichandran V, Saraswathi R, Mohanty B, Dhanaraj SK. Drug utilization studies - an overview. Int J Pharm Sci Nanotech. 2010;3(1):803-10. https://doi.org/10.37285/ijpsn.2010.3.1.2

2. Wettermark B, Elseviers M, Almarsdóttir AB, Andersen M, Benko $\mathrm{R}$, Bennie $\mathrm{M}$, et al. Introduction to drug utilization research. In: Elseviers M, Wettermark B, Almarsdóttir AB, Andersen M, Benko R, Bennie M, et al., editors. Drug utilization research: methods and applications. 1st ed. Wiley-Blackwell; 2016. p. 3-14.

3. StromBL, KimmelSE, HennessyS. Textbook of pharmacoepidemiology. Wiley Online Library; 2013.

4. Chatterjee S, Mandal A, Lyle N, Mukherjee S, Singh AK. Drug utilization study in a neonatology unit of a tertiary care hospital in eastern India. Pharmacoepidemiol Drug Saf. 2007;16(10):1141-5. https://doi.org/10.1002/pds.1469

5. Warrier I, Du W, Natarajan G, Salari V, Aranda J. Patterns of drug utilization in a neonatal intensive care unit. J Clin Pharmacol. 2006;46(4):449-55. https://doi. org/10.1177/0091270005285456 most commonly administered. Previous study by Warrier et al..$^{5}$ reported higher exposure to antibiotics like ampicillin (94.22\%) and cefotaxime. In general, high numbers and doses of antibiotics in infants, especially because of the potential for antibiotic resistance, should be prescribed more carefully.

The current study had some limitations. It was conducted in a short period of time, and it would have been better if it had lasted up to a year to prevent the seasonal variation of disease pattern. Data were collected from only one institute, and multicentric studies in this subject are needed. The patient case sheets were used for data extraction, which were the secondary source of data, and there was a possibility of bias.

\section{CONCLUSIONS}

This was a prospective study in a tertiary care hospital which was conducted for a period of 6 months to observe the utilization pattern of drug in NICU. Preterm delivery was one of the most important factors in the rate of hospitalization and treatment costs. Premature birth and resulting low birth weight were the main reasons for drug prescription. High administration of antibiotics is probably an area of concern and should be seriously considered.

\section{AUTHORS' CONTRIBUTIONS}

FN, SR: Conceptualization, Data curation, Formal Analysis, Funding acquisition, Investigation, Methodology. EMS, NBSM: Resources, Software, Supervision, Validation, Visualization, Writing - original draft, Writing - review \& editing.

6. Krzyżaniak N, Pawłowska I, Bajorek B. Review of drug utilization patterns in NICUs worldwide. J Clin Pharm Ther. 2016;41(6):61220. https://doi.org/10.1111/jcpt.12440

7. Al-Turkait A, Szatkowski L, Choonara I, Ojha S. Review of drug utilization studies in neonatal units: a global perspective. Int J Environ Res Public Health. 2020;17(16):5669. https://doi.org/10.3390/ ijerph17165669

8. Chourasia N, Surianarayanan P, Adhisivam B, Bhat BV. NICU admissions and maternal stress levels. Indian J Pediatr. 2013;80(5):380-4. https://doi.org/10.1007/s12098-0120921-7

9. Loudin S, Werthammer J, Prunty L, Murray S, Shapiro JI, Davies $\mathrm{TH}$. A management strategy that reduces NICU admissions and decreases charges from the front line of the neonatal abstinence syndrome epidemic. J Perinatol. 2017;37(10):1108-11. https:// doi.org/10.1038/jp.2017.101

10. Chawla D, Agarwal R, Deorari AK, Paul VK. Fluid and electrolyte management in term and preterm neonates. Indian J Pediatr. 2008;75(3):255-9. https://doi.org/10.1007/s12098-0080055-0 
11. Donge T, Bielicki JA, Anker J, Pfister M. Key components for antibiotic dose optimization of sepsis in neonates and infants. Front Pediatr. 2018;6:325. https://doi.org/10.3389/fped.2018.00325

12. Stempel DA, Durcannin-Robbins JF, Hedblom EC, Woolf R, Sturm LL, Stempl AB. Drug utilization evaluation identifies costs associated with high use of beta-adrenergic agonists. Ann Allergy Asthma Immunol. 1996;76(2):153-8. https://doi.org/10.1016/ s1081-1206(10)63415-1

13. Belén Rivas A, Arruza L, Pacheco E, Portoles A, Diz J, Vargas E. Adverse drug reactions in neonates: a prospective study. Arch Dis Child. 2016;101(4):371-6. https://doi.org/10.1136/ archdischild-2015-309396

14. Kumbhar AV, Halasawadekar NR, Ramanand SJ, Ramanand JB, Patil PT, Shah RD et al. Drug utilization study in a neonatal intensive care unit of a government tertiary care hospital in Western Maharashtra. Int J Basic Clin Pharmacol. 2018;7(8):1572. https:// doi.org/10.18203/2319-2003.ijbcp20183026
15. Choure MK, Jadhav RR, Padwal S. Drug utilization study in neonatal intensive care unit at rural tertiary care hospital. Asian J Pharm Clin Res. 2017;10(4):102. https://doi.org/10.22159/ajpcr.2017. v10i4.16111

16. Patel Brijal S, KubavatAmita R, Sondarva Divyesh B, Piparva Kiran G. Drug utilization study in neonatal intensive care unit at tertiary care hospital, Rajkot, Gujarat: a prospective study. World Journal of Pharmacy and Pharmaceutical Sciences. 2015;4(7):2034-42.

17. Gonçalves ACS, Reis AMM, Gusmão ACM, Bouzada MCF. Drug utilisation profile in the neonatal unit of a university hospital: a prospective observational study in Brazil. Int J Clin Pharm. 2015;37(4):645-55. https://doi.org/10.1007/s11096-015-0109-0

18. Neubert A, Lukas K, Leis T, Dormann H, Brune K, Rascher W. Drug utilisation on a preterm and neonatal intensive care unit in Germany: a prospective, cohort-based analysis. Eur J Clin Pharmacol. 2010;66(1):87-95. https://doi.org/10.1007/s00228009-0722-8 